\title{
Endobronchial sonography: feasibility and preliminary results
}

\author{
Thomas Hürter, Peter Hanrath
}

\begin{abstract}
Endobronchial sonography, a new ultrasound technique, has been evaluated for the assessment of normal lungs and bronchial carcinomas. The procedure was performed with ultrasound catheters, which were introduced into central and peripheral bronchi through the operating channel of fibreoptic bronchoscopes. The bronchial wall is highly echogenic and laminated. The lung parenchyma appears echo rich and patchy. Pulmonary arteries can be identified by the pulsatile changes and floating echoes within the echo free lumen. Echo poor bronchial carcinomas were detected in 69 out of 74 patients with endoscopically visible tumours and in 19 out of 26 patients with peripheral carcinomas. The correct implantation of metallic stents was facilitated by endobronchial sonography in nine patients. The sonographic examination carried no particular risk and caused little discomfort.
\end{abstract}

Transthoracic or transoesophageal sonographic approaches ${ }^{1-5}$ to the lungs and mediastinum are of limited value for the diagnosis of bronchial carcinoma. This is due to the interposition of air in lung parenchyma and bronchi between the transducer and the tumour. To overcome these diagnostic limitations we have assessed whether an intrabronchial approach using ultrasound catheters is useful. In this report the technical details and the sonomorphology of normal lungs and bronchial carcinomas are presented.

Medizinische Klinik I, Rheinisch-Westfälische Technische Höchschule Aachen, D-5100 Aachen, Germany T Hürter

P Hanrath

Reprint requests to: Dr T Hürter

Accepted 10 April 1992 gested bronchial carcinoma. All patients gave their written informed consent to the combined endoscopic-sonographic examination.

The endoscopy was performed after a subcutaneous injection of $15 \mathrm{mg}$ hydrocodone and $0.5 \mathrm{mg}$ atropine as an antitussive as well as intravenous sedation with $2 \cdot 5-5 \mathrm{mg}$ midazolam. For mucosal anaesthesia $10 \mathrm{ml}$ of $4 \%$ lignocaine was inhaled from an ultrasonic nebuliser. During inspection up to $10 \mathrm{ml}$ of $2 \%$ lignocaine was applied. The bronchoscopic examination started with the inspection of the central airways. Tumours were categorised endoscopically as central $(n=74)$, when there were typical signs of endobronchial $(n=56)$ or peribronchial $(\mathrm{n}=18)$ growth, or peripheral, when endoscopic inspection revealed no abnormalities $(n=26)$. Necropsy studies were performed in eight patients and the lungs investigated sonographically in a waterbath within 24 hours of death.

Endobronchial sonography was performed with 4.8 and $6.2 \mathrm{~F}$ catheters (Boston Scientific, Watertown, Massachusetts), which are flexible enough to be passed into all lobar segments via the operating channel of a fibreoptic bronchoscope (Olympus BF1T20D). These ultrasound catheters consist of a single element transducer, which is placed at the tip of a metal shaft (fig 1 ,

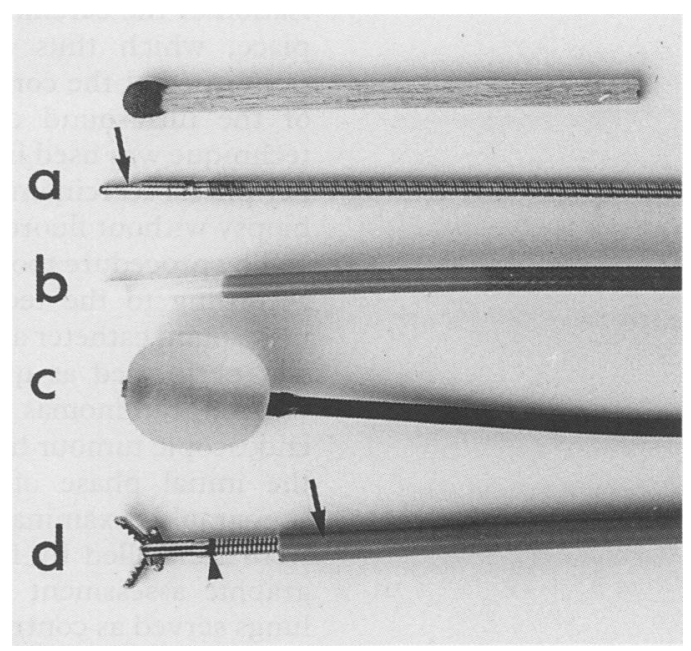

Figure 1 Ultrasound catheters for endobronchial sonography. The transducer ( $a$, arrow) rotates within a polyethylene sheath ( $b$ ) filled with water. $A$ water insufflated latex balloon (c) is used in large bronchi, while biopsy specimens from the peripheral lesions are accomplished by an $8 F$ guiding catheter (d, arrow) for accomplished by an $8 \mathrm{~F}$ guiding catheter (d, arrow)
the $4 \cdot 8 \mathrm{~F}$ ultrasound catheter and the flexible forceps (arrowhead). Top: a match to show the scale. 
Figure 2 Macroscopic appearance of a necropsy specimen showing normal lung tissue.

$P A-$ pulmonary artery; $B$-bronchus.

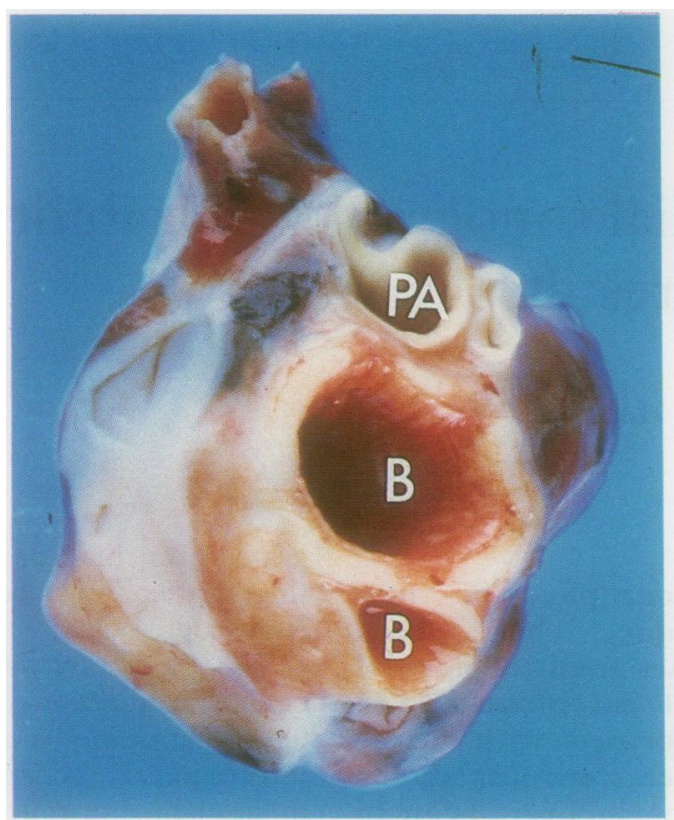

a), rotating within a polyethylene catheter sheath filled with sterile water (fig 1, b). Stenotic central bronchi and peripheral parts of the bronchial system were assessed with the ultrasound catheter alone. Good ultrasonic coupling between the mucosa and the ultrasound catheter was facilitated by bronchial secretions. In some cases only the instillation of $0.8 \%$ sodium chloride solution, as used in bronchoalveolar lavage, was necessary. The normal sized main, lobar, and segmental bronchi were assessed in the last eight patients with the help of a water filled latex balloon at the tip of the catheter (fig 1, c), which allowed the whole circumference to be visualised undisturbed by air. Peripheral biopsy specimens were obtained with a $4.8 \mathrm{~F}$ ultrasound catheter simultaneously passed into the tumour within an $8 \mathrm{~F}$ sheath through the working channel of a larger bronchoscope (Olympus BFXT20). After sonographic localisation of the carcinoma the sheath was left in place, which thus allowed a biopsy to be performed at the correct place after retraction of the ultrasound catheter (fig 1, d). This technique was used in the last six patients with peripheral carcinomas, making a successful biopsy without fluoroscopic control possible.

The procedure took from one to 20 minutes, according to the technique used. When the ultrasound catheter alone was used sonography was performed as quickly as forceps biopsy. Central carcinomas were assessed after the endoscopic tumour had been localised. During the initial phase of these investigations all sonographic examinations of peripheral lesions were controlled by fluoroscopy. In the sonographic assessment the healthy contralateral lungs served as controls.

The ultrasound frequency of the transducer is $20 \mathrm{MHz}$, the sector $360^{\circ}$ perpendicular to the longitudinal axis of the catheter. The maximum field of view is $5 \mathrm{~cm}$ in diameter under optimal conditions. The limit of the radial resolution is of the order of $200 \mu \mathrm{m}$. The two dimensional image is analysed in real time on a video monitor of the imaging console (DRF
1000, Diasonics, Milpitas, California). For contrast sonography of the pulmonary arteries $10 \mathrm{ml}$ oxypolygelatine is injected into the left or right cubital vein.

No serious complications occurred. In some cases the ultrasound catheter caused minimal bleeding, which stopped spontaneously. Patients sometimes noted more coughing during sonography, especially after water insufflation of the balloon.

\section{Results}

The bronchial wall is highly echogenic and has a unilaminar and a trilaminar structure in adjacent parts of the same bronchus. Trilaminar areas are composed of an inner echo dense layer followed by an intermediate echolucent zone of nearly the same diameter. These structures are surrounded by a thin echo dense band. Comparative in vitro studies showed that the three layered pattern corresponds to the regions with cartilage (figs 2 and 3 ), whereas the unilaminar structure is found in the intercartilagenous areas. Bronchial secretions appear echo free except for echo rich air bubbles moving within the lumen. Pulmonary arteries in close proximity to the bronchial wall can be identified in vivo by pulsatile changes of their diameter, spontaneous echoes within the echo free lumen, or microbubbles passing through the vessel after intravenous injection of an echo contrast medium. The artery wall is thin and echo rich. Both bronchi and pulmonary arteries are surrounded by lung parenchyma, which is characterised by its echo rich, patchy sonographic pattern.

Bronchial carcinomas of all histological types have an echo poor texture both in vivo and in vitro and thus can be differentiated from the highly echogenic normal bronchial wall and the

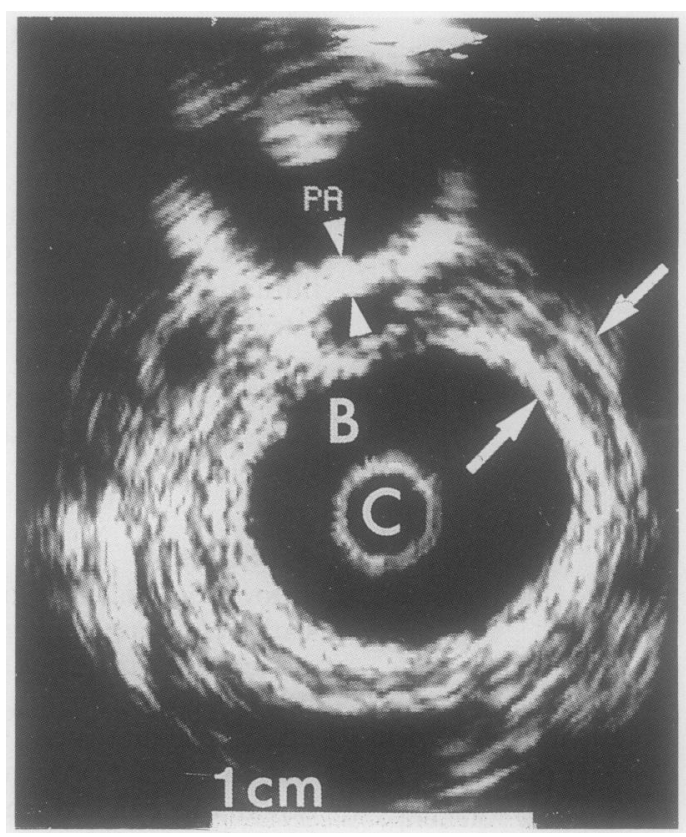

Figure 3 Ultrasound picture of the specimen shown in figure 2. The cup shaped pulmonary artery (PA) has an echo rich, thin layered, unilamellar wall (arrowheads), in contrast to the bronchial wall, which appears trilaminated (arrows) with an inner and outer echo rich layer and an echo poor lamina between. Both the water filled lumina of the pulmonary artery and bronchi are echo free. $C-$ catheter. 
Figure 4 Computed tomogram from a 58 year old patient showing a $2 \mathrm{~cm}$ nodule (arrow) in the left upper lobe.

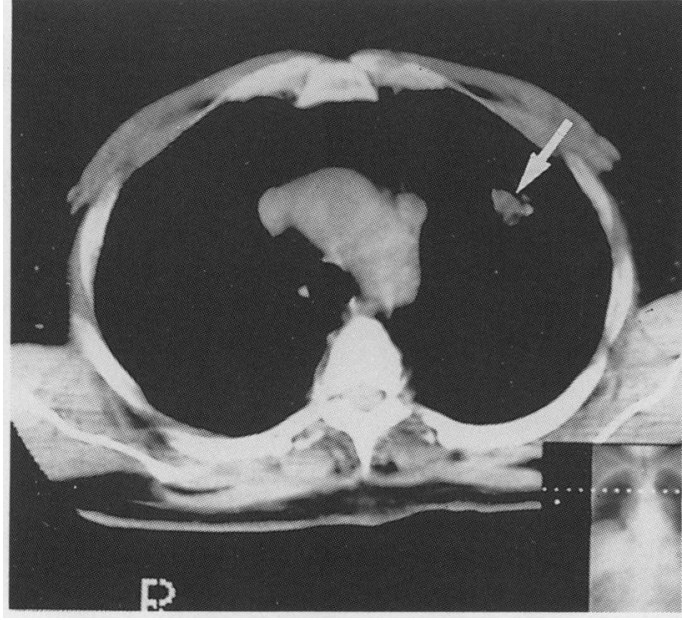

surrounding lung parenchyma (figs 4 and 5). Endobronchial sonography identifies endobronchial and peribronchial tumour growth as well as infiltration of the bronchial wall. Longitudinal and radial tumour extensions can be measured by retracting the catheter within the bronchus. Sixty nine out of 74 central carcinomas, which had been localised endoscopically, were identified by endobronchial sonography. In the remaining five patients peribronchial tumour growth had completely occluded the bronchial lumen, thus making passage and sonographic assessment impossible. Peripheral tumours, which could not be identified by bronchoscopy alone, were seen sonographically in 19 out of 26 cases. The remaining seven carcinomas were missed because the ultrasound catheter failed to come into close enough contact with the lesion. In all patients investigated no echo poor areas of tissue were found in contralateral lungs when radiograph and endoscopy had shown normal lung structurethat is, there were no false positive results.

In nine patients with peribronchial tumour growth self expandable metallic stents (Wallstent, Schneider, Zürich) were implanted for drainage of abscesses $(n=2)$, resolution of pneumonia $(n=2)$, or the relief of dyspnoea $(n=5)$. In all of these cases sonography was an adjunct to endoscopy because it allowed the preinterventional measurement of the length and diameter of the stenotic area, which could not be done by the fibreoptic bronchoscope.

Figure 5 Endobronchial sonogram from the patient of figure 4, showing the echo poor bronchial carcinoma (BC) as well as the echo rich lung parenchyma $(L P)$ and bronchial wall (arrowheads). An adenocarcinoma was diagnosed by endobronchial brushing.

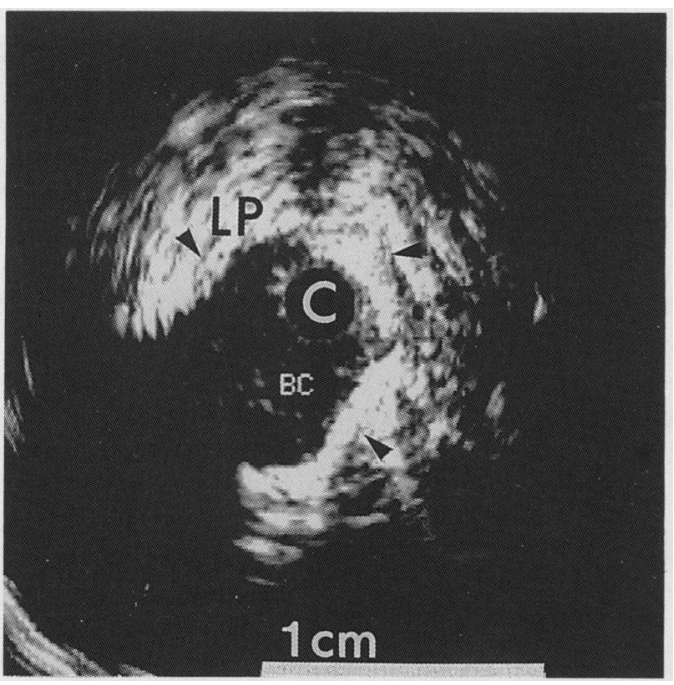

\section{Discussion}

The sonographic examination of bronchial carcinoma is still of limited value owing to the interposition of air within the bronchi and lung parenchyma between the transducer and the tumour. Thus both with the transthoracic and with the transoesophageal approach carcinomas are visible only in patients with tumours of the mediastinum or pleura..$^{1-5}$ The aim of this study was to test whether this limitation can be circumvented by an endoluminal approach. For this purpose we used an ultrasound catheter that was originally designed for vascular examinations. ${ }^{67}$ These small sized catheters were appropriate even in patients with bronchial stenosis or endoscopically invisible tumours.

To judge by our initial experience endobronchial sonography can be successfully performed in most patients during routine fibreoptic bronchoscopy. The method was well tolerated by the patients because it caused only mild discomfort. In addition, the technique was readily accepted by the investigators as it was simple and had no complications.

Endobronchial sonography allows the echo poor bronchial carcinoma and the highly echogenic normal bronchial wall or lung parenchyma to be differentiated, as shown in vitro and in vivo. Until now we have not had any experience with benign solid masses, which may contribute to false positive results. Endobronchial sonography definitely cannot distinguish between different cell types of lung cancer.

According to our preliminary results, endobronchial sonography may be an alternative to fluoroscopy for localising peripheral tumours. It can measure the length and diameter of bronchial stenoses that cannot be assessed by inspection or radiography. If computed tomography is not available, it may help to identify large vessels near tumours. Further studies are necessary to define its precise role here.

We wish to thank Dr J Ammon, director of the radiotherapy clinic, University Clinic Aachen, for providing the chest radiograph.

1 Izumi S, Tamaki S, Natori H, Kira S. Ultrasonically guided aspiration needle biopsy in disease of the chest. Am Rev Respir Dis 1982;125:460-4.

2 Kondo D, Imaizumi M, Abe T, Naruke T, Suemasu K. Endoscopic ultrasound examination for mediastinal lymph node metastases of lung cancer. Chest 1990;98: 586-93.

3 Pang JA, Tsang V, Hom BL, Metreweli C. Ultrasoundguided tissue-core biopsy of thoracic lesions with Trucut and Surecut needles. Chest 1987;91:823-8.

4 Wernecke K, Vassallo P, Pötter R, Lückener HG, Peters PE. Mediastinal tumors: sensitivity of detection with sonography compared with CT and radiography. Radiology 1990;175:137-43.

5 Yang PC, Luh KT, Sheu JC, Kuo SH, Yang SP. Peripheral pulmonary lesions: ultrasonography and ultrasonically guided aspiration biopsy. Radiology 1985;155:451-6.

6 Isner JM, Rosenfield K, Losordo DW, Kelly S, Palefski P, Langevin RE, et al. Percutaneous intravascular US as adjunct to catheter-based interventions: preliminary experience in patients with peripheral vascular disease. Radiology 1990;175:61-70.

7 Pandian NG, Weintraub A, Kreis A, Schwartz SL, Konstam MA, Salem DN. Intracardiac, intravascular, twodimensional, high-frequency ultrasound imaging of pulmonary artery and its branches in humans and animals. Circulation 1990;81:2007-12. 